\section{Targeting huntingtin}

Huntington's disease (HD) is caused by the expansion of a CAG repeat within exon 1 of the huntingtin (HTT) gene, resulting in an expanded polyglutamine stretch that forms insoluble aggregates associated with neuronal dysfunction and cell death. Because transcriptional dysregulation is part of the complex pathogenesis of $\mathrm{HD}$, histone deacetylases (HDACs) have been evaluated as therapeutic targets. HDAC4 is a transcriptional repressor that shuttles between the nucleus and cytoplasm and represses the transcription of genes with a role in neuronal cell death. HDAC4 is thought to self-aggregate through its glutamine-rich $\mathrm{N}$ terminus. Bates and colleagues now show that HDAC4 associates with mutant HTT in vivo in a polyglutamine-lengthdependent manner and colocalizes with cytoplasmic inclusions in the brains of HD mouse models. HDAC4 depletion inhibited cytoplasmic aggregate formation in HD mice, and this was accompanied by a pronounced restoration of synaptic function. In addition, knockdown of HDAC4 partially restored motor coordination and other neurological phenotypes and extended lifespan, thus suggesting a substantial contribution of cytoplasmic HTT aggregation to the pathology of HD. HDAC4 did not relocalize to the nucleus during disease progression, and HDAC4 knockdown had no effect on global transcriptional dysregulation and did not modulate nuclear HTT aggregation. Altogether, the data suggest a crucial role for cytoplasmic HTT aggregation in HD pathogenesis and identify HDAC4 as a promising therapeutic target. (PLOS Biol. doi:10.1371/journal.pbio.1001717, 26 November 2013)

$A H$

\section{Mind your pols and Qs}

RNA polymerase I ( $\mathrm{Pol} \mathrm{I}$ ) is the only nuclear RNA polymerase dedicated exclusively to synthesizing rRNA, which is transcribed in the nucleolus as a single 35S RNA precursor. Now, Kouzarides and colleagues describe a modification of histone $\mathrm{H} 2 \mathrm{~A}$ that is unique to the $35 \mathrm{~S}$ transcription unit and that has a Pol I-specific regulatory function. A survey of MS datasets to examine the prevalence of glutamine methylation within histones revealed a single site on Saccharomyces cerevisiae H2A, Q105, that was also detected on human H2A Q104. The Q105 methyltransferase activity was purified from yeast lysates and conclusively identified as the rRNA methyltransferase Nop1 by gene knockout; in vitro assays using recombinant protein confirmed the findings. Knockdown of Fibrillarin, the human Nop1 homolog, reduced H2A Q104 methylation in nucleoli of human cells. Transcription-inhibition assays showed that methylation of Q104 or Q105 requires active Pol I transcription. Intriguingly, Q105 is located in a region of $\mathrm{H} 2 \mathrm{~A}$ that is recognized by FACT, a nucleosomeremodeling complex that facilitates transcription elongation through chromatin. The authors show that methylation or alanine substitution of Q105 reduces H2A-peptide binding by FACT in vitro and that Q105A enhances transcription of reporters inserted into the yeast rRNA gene locus. Mutations that reduce FACT protein levels mimic the effects of Q105A, suggesting that methylation-responsive Q105-FACT interactions regulate Pol I activity within the rRNA transcription unit. Indeed, overexpressing Nop1 to increase Q105 methylation reduces FACT levels at the rRNA locus, thus revealing how glutamine methylation may modulate chromatin configuration to couple rRNA synthesis to the demands of cell growth. (Nature doi:10.1038/nature12819, 18 December 2013) BM

Written by Arianne Heinrichs, Stéphane Larochelle, Michelle Montoya \& Beth Moorefield

\section{Babysitting the clock}

Negative feedback is a conserved element in the maintenance of circadian rhythms. In Neurospora crassa, rhythmic oscillation is under the control of a central system in which the White Collar transcription-factor complex (WCC) stimulates expression of the clock protein FREQUENCY (FRQ). FRQ associates with the FRQ-interacting RNA helicase (FRH), and together they recruit factors that inactivate the WCC and subsequently promote the destruction of FRQ, thus resetting the cycle. Although FRH was known to stabilize FRQ and to stimulate the interaction between FRQ and the WCC, the molecular basis for FRH's activity in regulating periodicity remained poorly characterized. Dunlap and colleagues now expose an unexpected mechanism of action for FRH that is independent of its enzymatic activity and sheds light on the hitherto-underappreciated characteristics of FRQ as an intrinsically disordered protein (IDP). First, the authors demonstrated that although the helicase activity of FRH is required for normal growth, the clock function of FRH could be fully restored by a mutant devoid of helicase activity. They then showed that FRH exhibits many of the biochemical characteristics of an IDP and that its clock function probably lies in its ability to act as a 'nanny' protein to protect FRQ from premature degradation and/or spurious interactions. Although it is yet to be determined whether similar interactions are involved in regulating other circadian oscillators, the findings highlight the emerging concept that the functional properties of IDPs might often be in stabilizing interactions with cellular factors that carry distinct cellular roles. (Mol. Cell doi:10.1016/j.molcel.2013.11.005, 5 December 2013) SL

\section{TALlying lipid-protein interactions}

Interactions between lipid membranes and peripheral membrane proteins are essential to many physiological processes. Assays that use liposomes as artificial membranes are widely used because they allow for the creation of lipid mixtures that closely approximate native membrane environments. However, many of these assays require large quantities of lipids and purified proteins, and it is often difficult to scale up lipid production protocols. In addition, once produced, lipids are stable in biological buffers for only a few days. Now, Gavin and colleagues have devised a liposome microarray-based assay (LiMA) that uses liposomes assembled from lipid mixtures that have been soaked into a thin agarose layer (TAL). The authors apply spots of lipid mixtures composed of signaling lipids, such as glycerophospholipids and sterols, the carrier lipid POPC and a fluorescently labeled lipid, such as PE-Atto 647, on the TAL. The lipid-spotted TAL could be stored under argon for at least 2 months. For the binding assays, the TAL was coupled to a microfluidic chamber, and a GFP-tagged lipid-binding protein was introduced. The authors' final device contained four different chambers, thus permitting the examination of four different signaling proteins interacting with 30 different lipid membranes. After incubation, imaging was performed on an automated fluorescence microscopy platform, and the number of GFP-tagged proteins bound per unit of membrane surface area was calculated. LiMA can be used to detect cooperative binding events and discrete changes in binding affinities brought about, for example, by protein mutation. It has the potential to be scaled to proteomic or lipidomic analyses and can make use of the readily available cell lines producing GFP fusions. (Nat. Methods doi:10.1038/ nmeth.2734, 24 November 2013) MM 